\title{
Descenso transanal endorrectal De la Torre como tratamiento de Enfermedad de Hirschsprung, Hospital Escuela, 2013-2015
}

\author{
Transanal endorectal pull-through De la Torre as treatment for Hirschsprung's disease, \\ University Hospital, 2013-2015. \\ David Alexander Montoya-Reales, ${ }^{1}$ Josué Fernando Rodas Andino, ${ }^{2}$ \\ Roberto Antonio Martínez Quiroz. ${ }^{3}$
}

\begin{abstract}
'Medico General, Egresado de la Facultad de Ciencias Médicas, Universidad Nacional Autónoma de Honduras, año 2014. ${ }^{2}$ Medico General, Egresado de la Facultad de Ciencias Médicas, Universidad Nacional Autónoma de Honduras, año 2015. ${ }^{3}$ Cirujano Pediatra; Servicio de Cirugía Pediátrica, Departamento de Pediatría, Hospital Escuela.
\end{abstract}

\begin{abstract}
RESUMEN. Antecedentes: La enfermedad de Hirschsprung (EH) es causa de obstrucción intestinal baja en neonatos. En 1998, De la Torre y Ortega publicaron una importante modificación a la técnica de Soave, realizando un descenso transanal endorrectal. Objetivo: Describir la experiencia en el tratamiento de la Enfermedad de Hirschsprung mediante la técnica descrita por De la Torre, Hospital Escuela, Tegucigalpa, 2013-2015. Metodología: Estudio descriptivo transversal. Se revisaron expedientes clínicos y se registró información sobre características sociodemográficas y clínicas de los pacientes. Los resultados se presentan como frecuencias y porcentajes de las variables estudiadas. La información personal de los pacientes se manejó confidencialmente. Resultados: Se identificó un total de 26 casos intervenidos en el periodo del estudio. El 73.1\% (19) pertenecía al sexo masculino, 46.2\% (12) se diagnosticó antes de 12 meses de vida; sin enfermedades asociadas. La biopsia diagnosticó la enfermedad en el 100\% de los casos. La longitud del colon resecado fue de $10-20 \mathrm{~cm}$ en $88.5 \%$ (23), con un tiempo quirúrgico de $4-5$ horas $69.2 \%$ (18). El 19.2\% (5) presentó complicaciones. Se inició alimentos en menos de 5 días en $96.1 \%$ (25), la estancia hospitalaria fue menor a 7 días en $88.5 \%$ (23). Discusión: El descenso endorrectal transanal es una operación segura con pocas complicaciones operatorias y postoperatorias. En este estudio, la estancia hospitalaria y el tiempo hasta la alimentación oral completa fueron más cortos que los procedimientos convencionales lo que generó menores costes hospitalarios. Consideramos que ésta es la técnica de elección para enfermos con la Enfermedad de Hirschsprung.

Palabras clave: Descenso transanal endorrectal, Enfermedad de Hirschsprung, Megacolon congénito.
\end{abstract}

\section{INTRODUCCIÓN}

Tradicionalmente el tratamiento de la enfermedad de Hirschsprung (EH) o megacolon agangliónico (MA) implicaba la necesidad de realizar varias intervenciones quirúrgicas, con sus correspondientes anestesias generales e ingresos, que terminaban después del año de vida. En el momento del diagnóstico se realizaba una colostomía, para en un segundo ingreso realizar la resección del segmento agangliónico y el descenso del intestino normalmente inervado y en una tercera intervención practicar el cierre de la colostomía. ${ }^{1}$ El manejo quirúrgico ha evolucionado de dos o tres etapas realizadas tradicionalmente a un descenso en una sola etapa. ${ }^{2}$ El descenso endorrectal descrito por Soave en 1964 es una de las técnicas clásicamente utilizadas para el tratamiento de esta enfermedad. Boley, en el mismo año, describió una modificación ampliamente aceptada consistente en realizar la anastomosis en el mismo tiempo quirúrgico por vía perineal.

Recibido: 3-10-2018; Aceptado para publicación 20-3-2019

Dirección para correspondencia: Dr. David Alexander Montoya Reales.

Correo electrónico: montoyareal7485@hotmail.com

Conflictos de interés. Los autores no tienen conflictos de interés que declarar en relación a este estudio.

DOI: https://doi.org/10.5377/rmh.v87i1.11928
En 1995 Georgeson introdujo dos modificaciones a la técnica de Soave-Boley: el abordaje laparoscópico para el tiempo abdominal y una disección transanal más extensa de forma que realiza el manguito mucoso por esta vía. ${ }^{1}$ Después fue descrito el abordaje transanal por De la Torre y Ortega ${ }^{3}$ y Langer et al. ${ }^{4}$

De la Torre y Ortega publicaron una importante modificación consistente en utilizar únicamente la vía transanal para realizar la mucosectomia rectal, la resección del segmento agangliónico, el descenso y la anastomosis del colon normogangliónico. ${ }^{1}$ Sus resultados son comparables o mejores a las técnicas tradicionales, debido a que elimina la incisión abdominal y las complicaciones de la laparotomía convencional. Por otra parte, disminuye la estadía hospitalaria y los costes en sentido general. Teóricamente se mantiene la integridad del esfínter anal externo y las posibilidades de lesión de nervios pélvicos son menores. ${ }^{5}$ La resección quirúrgica del intestino inervado anormalmente es esencial para evitar complicaciones que amenazan la vida y proveer un paso de las heces sin obstrucción. Sin embargo, una proporción significativa de pacientes continúa teniendo defectos posoperatorios persistentes en la función intestinal. Variando en grados de incontinencia fecal, constipación y colitis afectando a la mitad de los pacientes, impactando significativamente en la calidad de vida y causando morbilidad a 
largo tiempo, ${ }^{6,7}$ El presente estudio se realizó con el objetivo de describir la experiencia en el tratamiento de la Enfermedad de Hirschsprung mediante la técnica descrita por De la Torre en el Hospital Escuela, Tegucigalpa, en el periodo 2013-2015.

\section{METODOLOGÍA}

Estudio descriptivo transversal sobre pacientes menores de 18 años de edad, de ambos sexos, con diagnóstico de Enfermedad de Hirschsprung, intervenidos quirúrgicamente con la técnica de descenso transanal endorectal de la Torre, atendidos en el Servicio de Cirugía Pediátrica, Departamento de Pediatría, Hospital Escuela, durante el periodo de enero 2013 a diciembre 2015.

La fuente de la información fue el expediente clínico. Se registró la información en un instrumento diseñado para tal efecto, incluyendo características sociodemográficas (edad de diagnóstico, sexo, lugar de procedencia, situación socioeconómica), antecedentes (edad de la madre durante el embarazo, control prenatal, número de hijos) y clínicas (malformaciones congénitas asociadas, tiempo entre el diagnóstico y la operación, biopsia preoperatoria, número de biopsias transoperatorias realizadas, estudios de imagen previos a la cirugía, longitud del colon resecado, tiempo de la cirugía, tiempo entre la cirugía y el inicio de la vía oral, complicaciones post operatorias, tiempo de estancia hospitalaria).

La información recopilada fue digitada en una base de datos electrónica diseñada en Epilnfo Versión 7.1.5 (CDC, Atlanta EUA). Una vez realizado el control de calidad de la base de datos y limpieza final, se utilizó el módulo análisis, parte del mismo software para generar un reporte estadístico compuesto por cuadros simples. La información personal de los pacientes se manejó de forma confidencial.

\section{RESULTADOS}

En el periodo del estudio se intervinieron con la técnica descrita por De la Torre 26 niños con diagnóstico de Enfermedad de Hirschsprung. Todos fueron incluidos en el estudio. En el Cuadro 1 se presentan algunas características sociodemográficas distribuidas por sexo. Los pacientes en su mayoría nacieron en Francisco Morazán 38.5\% (10), Comayagua con 23.1\% (6) y Olancho con $15.4 \%$ (4).

En cuanto a los antecedentes, la edad de la madre de los pacientes fue menor de 20 años en $23.1 \%$ (6) de los casos, entre 20 y 29 años en $15.4 \%$ (4), entre 30 y 39 años en $11.5 \%$ (3) y mayor de 40 años en $7.7 \%$ (2). En $42.3 \%$ (11) de los casos esta información no fue consignada. En el $57.7 \%$ (15) de los casos se registró información sobre controles prenatales, $15.4 \%$ (4) no se realizó control prenatal y en $26.9 \%$ (7) esta información no fue consignada. En el $34.6 \%$ (9) de los casos, el paciente era el primer hijo de la pareja, en el $15.4 \%$ (4) el segundo hijo y en $26.9 \%$ (7) de los casos esta información no fue consignada.
En cuanto a las características clínicas, ninguno de los pacientes tenía enfermedades asociadas. Se realizó colon por enema al $100 \%$ de los pacientes. En el Cuadro 2 se presentan los datos quirúrgicos distribuidos por sexo, incluyendo tiempo entre el diagnóstico y la operación, biopsia pre-operatoria, número de biopsias trans-operatorias realizadas, longitud del colon resecado, tiempo de la cirugía, tiempo entre la cirugía y el inicio de la vía oral. Dentro de los datos post-quirúrgicos, la estancia hospitalaria fue menor a 7 días en $88.5 \%$ (23), el $19.2 \%$ (5) presentó complicaciones. Las complicaciones incluyeron colitis (1), infecciones (2) y otras complicaciones (2).

Cuadro 1. Datos sociodemográficos de los pacientes operados con la técnica de descenso transanal endorrectal De la Torre y su distribución por sexo, Hospital Escuela, Tegucigalpa, 2013-2015, n=26.

\begin{tabular}{lcc}
\hline \multirow{2}{*}{ CARACTERISTICAS } & \multicolumn{2}{c}{ SEXO } \\
& $\begin{array}{c}\text { Masculino } \\
\mathbf{N}(\%)\end{array}$ & $\begin{array}{c}\text { Femenino } \\
\mathbf{N}=7\end{array}$ \\
& $\mathbf{N}(\%)$ \\
\hline Edad del diagnóstico (años) & & \\
$<1$ & $8(42.1)$ & $4(57.1)$ \\
$1-5$ & $6(31.6)$ & $3(42.9)$ \\
$6-10$ & $3(15.8)$ & $0(0.0)$ \\
$11-15$ & $2(10.5)$ & $0(0.0)$ \\
Condición socioeconómica & & \\
Pobre & $19(100.0)$ & $7(100.0)$ \\
\hline
\end{tabular}

Cuadro 2. Datos quirúrgicos de los pacientes operados con la técnica de descenso transanal endorrectal De la Torre y su distribución por sexo, Hospital Escuela, Tegucigalpa, 2013-2015, n=26.

\begin{tabular}{lcc}
\hline \multirow{2}{*}{ CARACTERISTICAS } & \multicolumn{2}{c}{ SEXO } \\
& Masculino $=19$ & Femenino \\
& $\mathbf{N}(\%)$ & $\mathbf{N}(\%)$ \\
\hline $\begin{array}{l}\text { Tiempo entre el diagnóstico y la } \\
\text { operación (meses) }\end{array}$ & & \\
$<1$ & $4(21.1)$ & $2(28.6)$ \\
$1-6$ & $6(31.5)$ & $2(28.6)$ \\
$7-12$ & $5(26.3)$ & $2(28.6)$ \\
$>12$ & $4(21.1)$ & $1(14.2)$ \\
Biopsia pre-operatoria & & \\
Si & $19(100.0)$ & $7(100.0)$ \\
Número de biopsias trans-operatoria & & \\
1 & $16(84.2)$ & $7(100.0)$ \\
2 & $3(15.8)$ & $0(0.0)$ \\
Longitud colon resecado (cm) & & \\
$10-20$ & $16(84.2)$ & $7(100.0)$ \\
$>20$ & $3(15.8)$ & $0(0.0)$ \\
Tiempo de la Cirugía (horas) & & \\
$2-3$ & $1(5.3)$ & $0(0.0)$ \\
$4-5$ & $14(73.7)$ & $4(57.1)$ \\
$>5$ & $4(21.0)$ & $3(42.9)$ \\
Tiempo entre cirugía e inicio de la vía & & \\
oral (días) & & \\
$<5$ & $18(94.7)$ & $7(100.0)$ \\
$\mathbf{5 - 1 0}$ & $1(5.3)$ & $0(0.0)$ \\
\hline
\end{tabular}




\section{DISCUSIÓN}

En 1886 el Profesor de Pediatría Harald Hirschsprung presentó su casuística de recién nacidos con estreñimiento que fallecieron. Señaló que las autopsias mostraban el colon transverso y descendente con gran dilatación e hipertrofia, pero que el recto y sigmoides no estaban afectados. ${ }^{8} \mathrm{La}$ EH es el desorden de motilidad intestinal congénita más común, caracterizada por la ausencia de células ganglionares en los plexos mientérico y submucoso (Auerbach y Meissner, respectivamente) del intestino distal, que resulta en la ausencia de peristalsis en el segmento afectado y el desarrollo de obstrucción intestinal funcional. Tiene una incidencia de 1/5.000-1/7.000 nacimientos, siendo más frecuente en varones que en mujeres. Puede estar asociado con otros síndromes médicos ( $18 \%$ de los casos), de los cuales más del $90 \%$ corresponde con el síndrome de Down. ${ }^{6,9-11}$ Los resultados de este estudio demuestran que el sexo masculino es el más afectado, resultados similares encontrados en otros estudios. ${ }^{12-15}$

La etiología sigue siendo no del todo comprendida. La mitad de los casos familiares y $15 \%$ de los casos esporádicos son asociados con mutaciones del gen que inactiva el receptor RET para tirosina quinasa en el cromosoma $10 q .{ }^{16}$ Los factores estudiados en nuestro estudio fueron el nivel socioeconómico donde el $100 \%$ de los pacientes eran pobres, otro factor fue la edad materna la cual no tuvo representación estadística, otro factor fueron los controles prenatales donde solo el $57.69 \%$ se lo realizaron. Esta condición se confina al colon rectosigmoide en el $75.0 \%$ de los pacientes afectados. En el $17.0 \%$ de los casos, se observa que el segmento agangliónico se extiende desde el recto, el colon sigmoide y el colon hasta la parte del colon transverso. La forma más rara de la enfermedad con el curso clínico más grave es la aganglionosis colónica total, que se observa en el $8.0 \%$ de los pacientes. ${ }^{17}$

El diagnóstico prenatal de la EH es raro, y generalmente se debe a una enfermedad colónica total que resulta en hallazgos de obstrucción intestinal fetal por ultrasonido (USG). La mayoría de los pacientes afectados se presentan durante el período neonatal con distensión abdominal, vómitos biliosos e intolerancia alimentaria. El retraso en el paso del meconio más allá de las primeras 24 horas está presente en aproximadamente el $90.0 \%$. Ocasionalmente, la perforación cecal o apendicular puede ser el evento inicial. ${ }^{18,19} \mathrm{El} 46.2 \%$ de nuestros pacientes fue diagnosticado durante el primer año de vida. No se encontraron malformaciones asociadas en esta casuística.

El diagnóstico oportuno y su manejo evitan las potenciales complicaciones de pacientes con EH. Entre los estudios iniciales por realizar, se encuentran los enemas contrastados, la manometría rectal y la biopsia rectal. ${ }^{20}$ En este estudio se encontró que se realizó colon por enema al $100.0 \%$ de los pacientes. La biopsia rectal se considera el estándar de oro para el diagnóstico de la HD. La presencia de células ganglionares en los plexos nerviosos submucoso y muscular excluye la enfermedad, por lo tanto, para hacer el diagnóstico es esencial que se obtenga una muestra adecuada. ${ }^{21,22}$ La biopsia rectal por succión, debido a su relativa facilidad y baja morbilidad, se ha convertido en la herramienta de diagnóstico más establecida para la EH. Sin embargo, en ocasiones se requieren biopsias rectales de espesor total. ${ }^{23}$ En este estudio se realizó biopsia rectal preoperatoria en el $100 \%$ de los pacientes.

La longitud del colon resecado en el $88.5 \%$ (23) fue de $10-20 \mathrm{~cm}$ y en $11.5 \%$ (3) se resecó más de $20 \mathrm{~cm}$. Datos que difieren con los encontrados en un estudio realizado en México donde el tamaño del segmento resecado en el $28.6 \%$ (6) fue de $10-20 \mathrm{~cm}, 57.1 \%$ (12) midió $21-30 \mathrm{~cm}$, y en $14.3 \%$ (3) resecó de $31-40 \mathrm{~cm} .{ }^{13}$ También difieren de uno realizado en Costa Rica en donde la longitud del segmento intestinal comprometido fue ultracorta en 12 pacientes, corta en 60 casos, larga en 23, y 7 casos presentaron megacolon total. ${ }^{20}$

Dado los numerosos avances en la cirugía, los resultados postoperatorios son satisfactorios, pero algunos pacientes mantienen disfunciones intestinales, como estenosis, incontinencia, ensuciamiento, distensión abdominal, constipación, diarrea y colitis, y en algunos casos, una combinación de ellos, ${ }^{24,25}$ con importantes consecuencias en las esferas personal, social y profesional que pueden reflejarse en la edad adulta. ${ }^{26} \mathrm{La}$ frecuencia de incontinencia oscila entre 0.0 a $58.0 \%$ y la de estreñimiento de 3.0 a $28.0 \% .{ }^{27} \mathrm{El}$ desarrollo de colitis en pacientes con EH constituye una importante causa de morbi-mortalidad. Puede presentarse como la primera manifestación clínica de la $\mathrm{EH}$ o durante su curso evolutivo antes o incluso años después de la resección del segmento agangliónico. La colitis es conocida como una complicación severa tras el tratamiento quirúrgico de la $\mathrm{EH}$, con una importante morbilidad y mortalidad. ${ }^{28} \mathrm{Se}$ reconoce una incidencia en hasta el $58 \%$ de los pacientes con un promedio del 28.5\%. ${ }^{29}$ En nuestro estudio, $19.2 \%$ (5) presentó complicaciones, de las cuales Colitis representó el 20.0\% (1). La estancia hospitalaria fue menor a 7 días $88.5 \%$ (23) y la vía oral se inició en menos de 5 días en el $96.1 \%$. Estos datos son similares a los informados en un estudio realizado en México en donde en las primeras 72 horas $90.5 \%$ reinició la vía oral y $47.6 \%$ se egresó. ${ }^{13}$

En conclusión, en este estudio sobre una casuística de 26 pacientes, una muestra pequeña, se puede apreciar el buen desenlace de esta técnica. En los casos descritos, el descenso endorrectal transanal ofreció un excelente abordaje para los niños con enfermedad de Hirschsprung. Fundamentados en estos resultados, recomendamos que esta técnica pueda ser usada por cualquier cirujano pediatra experimentado para el manejo de casos con la Enfermedad de Hirschsprung.

\section{CONTRIBUCIONES}

DAMR, FJRA y RAMQ contribuyeron en igual proporción en concebir y diseñar el estudio, en el manejo y recolección de datos, así como en la redacción del artículo. Todos los autores atendieron las recomendaciones editoriales y todos aprobaron la versión final del manuscrito. 


\section{REFERENCIAS}

1. Juliá V, Castañón M, Tarrado X, Pinzón J, Morales L. Descenso endorectal transanal exclusivo para el tratamiento de la enfermedad de Hirschsprung. Cir Pediatr. 2004; 17(2): 85-88

2. Nasr A, Haricharan RN, Gamarnik J, Langer JC. Transanal pullthrough for Hirschsprung disease: Matched case-control comparison of Soave and Swenson techniques. J Pediatr Surg. 2014; 49(5): 774-776.

3. De la Torre-Mondragon L, Ortega-Salgado JA. Transanal endorectal pullthrough for Hirschsprung's disease. J Pediatr Surg 1998; 33(8): 1283-6.

4. Langer J, Minkes R, Mazziotti M, Skinner M, Winthrop A. Transanal onestage Soave procedure for infants with Hirschsprung's disease. J Pediatr Surg. 1999; 34(1):148-51.

5. Hernández H, Vialat V, Labrada E, Navarro A. Evolución clínica de los pacientes operados de enfermedad de Hirschsprung por técnicas transanales. Rev Cubana Pediatr. 2012; 84(2): 216-24.

6. Neuvonen M, Kyrklund K, Lindahl H, Koivusalo A, Rintala R, Pakarinen M. A population-based, complete follow-up of 146 consecutive patients after transanal mucosectomy for Hirschsprung disease. J Pediatr Surg. 2015; 50(10):1653-8

7. Stensrud KJ, Emblem R, Bjørnland K. Anal endosonography and bowel function in patients undergoing different types of endorectal pull-through procedures for Hirschsprung disease. J Pediatr Surg. 2015; 50(8):1341-6.

8. De la Torre Mondragón L. Enfermedad de Hirschsprung. Mitos y realidades a 120 años de su descripción. Acta Pediatr Mex 2008; 29(3):139-46.

9. Ruiz Aja E, Vega Hernández L, Martínez Ezquerra N, De Diego García E, Pérez Marrodan A, López Álvarez Buhilla P. Características genéticas, poblacionales y fenotípicas de pacientes con enfermedad de Hirschsprung. Cir Pediatr 2012; 25: 135-39.

10. Vega Hernández L, Castaño González L, Belar Beitia O, Ruiz Aja E, Martinez Ezquerra N, Lopez Alvarez-Buhilla P. Estudios de polimorfismos asociados a la enfermedad de Hirschsprung. Cir Pediatr. 2011; 24: 131-36.

11. Luis LA, Encimas JL, Avila LF, Andres AM, Burgos L, Fernández A, et al. Enfermedad de Hirschsprung: enseñanzas de los últimos 100 casos. Cir Pediatr 2006; 19: 177-81.

12. Chams AA, Mejía FJ, Lince LF. Comparación de resultados entre las técnicas de Soave y el descenso trans-anal endo-rectal en el manejo de la enfermedad de Hirschsprung 2005-2007. CIRUPED. 2011; 1(1): 24-29.

13. Moreno-Denogean B, Barraza-León A, Noriega-Aldana JM, Carballo-Toledo JL. Descenso endorrectal por vía transanal. 7 años de experiencia. Rev Mex Cir Ped. 2014; 18(2), 71 - 78.

14. Ali KA. Transanal endorectal pull-through for Hirschsprung's Disease during the first month of life. Ann Pediatr Surg. 2010; 6(2), 81-88.

15. Al-Jazaeri A, Al-Shanafey S, Zamakhshary M, Al-Jarbou W, Hajr E, Breakeit $\mathrm{M}$, et al. The impact of variation in access to care on the management of Hirschsprung disease. J Pediatr Surg. 2012; 47(5):952-5.

16. Delgado Monge A. Enfermedad de Hirschsprung: diagnóstico y manejo en niños y adultos. Rev Méd Costa Rica Centroam. 2016; 73(620): 687 - 691.

17. Xia X, Li N, Wei J, Zhang W, Yu D, Zhu T, et al. Single-incision laparoscopic versus conventional laparoscopic surgery for Hirschsprung's disease: A comparison of medium-term outcomes. J Pediatr Surg. 2016; 51(3):440-3.

18. Langer JC. Hirschsprung disease. In: Holcomb III George W, Murphy Patrick J, Ostlie Daniel J, editors. Ashcraft's pediatric surgery. 6th ed. New York: Saunders Elsevier; 2014: 474-491.

19. Meinds RJ, Eggink MC, Heineman E, Broens PM. Dyssynergic defecation may play an important role in postoperative Hirschsprung's disease patients with severe persistent constipation. Analysis of a case series. J Pediatr Surg. 2014; (49): 1488-92.

20. García-Arias F, Ceciliano-Romero N. Análisis del manejo quirúrgico de la enfermedad de Hirschsprung en el Hospital Nacional de Niños Dr. Carlos Sáenz Herrera, durante el periodo 2000-2010. Acta méd costarric. 2013; 55(2): 87-91.

21. Stewart CL, Kulungowski AM, Tong S, Langer JC, Soden J, Sømme S. Rectal biopsies for Hirschsprung disease: Patient characteristics by diagnosis and attending specialty. J Pediatr Surg. 2016; 51(4):573-6.

22. Neves Romaneli MT, Ribeiro AF, Bustorff-Silva JM, Carvalho Lomazi EA. Hirschsprung's disease - Postsurgical intestinal dysmotility. Rev Paul. Pediatr. 2016; 34(3):388-92

23. Teitelbaum DH, Coran AG. Hirschsprung disease. In: Spitz L, Coran A, editors. Operative Pediatric Surgery. 7th ed. Boca Ratón, Florida: CRC Press; 2013: p.560-581.

24. Bazo M, Bailez M. Calidad de vida relacionada con la salud en niños y adolescentes con cirugía de enfermedad de Hirschsprung y malformaciones anorrectales. Arch Argent Pediatr. 2013; 111(1):37-44.

25. Guerra J, Wayne C, Musambe T, Nasr A. Laparoscopic-assisted transanal pull-through (LATP) versus complete transanal pull-through (CTP) in the surgical management of Hirschsprung's disease. J Pediatr Surg. 2016; 51(5): 770-774

26. Mathias AL, Tannuri AC, Ferreira MA, Santos MM, Tannuri U. Validation of questionnaires to assess quality of life related to fecal incontinence in children with anorectal malformations and Hirschsprung's disease Rev Paul Pediatr. 2016; 34(1):99-105.

27. Fernández M, Sánchez J, Martínez I, Reyes R, Cabrejos K, Rojas J, et al. Resultados funcionales en pacientes con enfermedad de Hirschsprung intervenidos mediante las técnicas de Duhamel y De la Torre. Cir Pediatr 2013; 26: 183-188

28. Vega N, Álvarez V, López A, Montalvo C, Oviedo M, Raposo L. Episodios de enterocolitis postoperatorios en pacientes intervenidos de enfermedad de Hirschsprung. Cir Pediatr. 2014; 27(2): 84-88.

29. Castañeda S, García A, Jaimes P, Jaramillo L, Perilla M, Mendez M, et al. Enterocolitis asociada a enfermedad de Hirschsprung. Experiencia en un Hospital Universitario Pediátrico. Cir Pediatr. 2014; 27(2): 78-83.

ABSTRACT. Background: Hirschsprung's disease (HD) is a common cause of lower bowel obstruction in neonates. In 1998, de la Torre and Ortega published an important modification of the Soave technique. Objective: To describe the experience in the treatment of Hirschsprung's disease with the technique described by De la Torre, Hospital Escuela, Tegucigalpa, 2013-2015. Methodology: A descriptive cross-sectional study. Clinical records were reviewed and information on sociodemographic and clinical characteristics of patients was recorded. The results are presented as frequencies and percentages of the variables studied. Patients' personal information was handled confidentially. Results: A total of 26 surgically intervened cases were identified during the study period. The $73.1 \%$ (19) male, $46.2 \%$ (12) were diagnosed before 12 months of life; without associated diseases. The biopsy diagnosed the disease in $100 \%$ of cases. The length of the resected colon was $10-20 \mathrm{~cm}$ in $88.5 \%(23)$, with a surgical time of $4-5$ hours $69.2 \%(18)$. The $19.2 \%$ (5) had complications. Food was started in less than 5 days in $96.1 \%(25)$, hospital stay was less than 7 days in $88.5 \%(23)$. Discussion: Transanal endorectal pull-through with a short cuff is a safe operation with low incidence of operative and postoperative complications. Hospital stay and time to full enteral feeding was significantly shorter than following conventional procedures; this associates with lower hospital costs. We believe this is the treatment of choice for patients with Hirschsprung disease.

Keywords: Congenital Megacolon, Hirschsprung disease, Transanal endorectal pull-through. 